\title{
Platelet-Rich Plasma Pretreatment on Grit-Blasted Titanium Alloy for Enhanced Osteogenic Differentiation of Human Adipose-Derived Stem Cells
}

\author{
Seong Hwa Hong, MD, Jinwoo Nam, Hee Joong Kim, MD, Jeong Joon Yoo, MD \\ Department of Orthopedic Surgery, Seoul National University College of Medicine, Seoul, Korea
}

\begin{abstract}
Background: Adequate bone formation around titanium alloy implants is integral to successful implantation surgery. Stem cellcoated implants may accelerate peri-implant bone formation. This study investigates the effect of platelet-rich plasma (PRP) pretreatment on a titanium-alloy surface in terms of proliferation and osteogenic differentiation of human adipose-derived stem cells (hADSCs).

Methods: Allogenic leukocyte-depleted PRP was obtained from blood supernatants. The hADSCs were isolated from thigh subcutaneous fat tissue. Grit-blasted titanium plugs were used in two different groups. In one group, $200 \mu \mathrm{L}$ of PRP was added to the grit-blasted titanium plugs. The hADSCs were seeded in two groups: grit-blasted titanium plugs with or without PRP. The number of hADSCs was measured after 4 hours, 3 days, and 7 days of culture using Cell Counting Kit-8. Osteogenesis of hADSCs was measured by using an alkaline phosphatase activity assay on days 7 and 14, and a calcium assay on days 14 and 21. Osteogenic gene expression was measured by using reverse transcription polymerase chain reaction analysis of alkaline phosphatase, osteocalcin, and type I collagen mRNA. The microscopic morphology of grit-blasted titanium plugs with or without PRP was examined with a field-emission scanning electron microscope using a JSM-7401F apparatus on days 1 and 7.

Results: Proliferation and osteogenic differentiation of hADSCs were found to be significantly higher on the grit-blasted titanium alloy preprocessed with PRP than the same alloy without pretreatment. Furthermore, a structural fibrillar mesh developed compactly on the grit-blasted titanium alloy with the PRP pretreatment.

Conclusions: Our results demonstrate that a hADSC-based approach can be used for tissue-engineered peri-implant bone formation and that PRP pretreatment on the grit-blasted titanium alloy can improve proliferation and osteogenic differentiation of hADSCs.
\end{abstract}

Keywords: Platelet-rich plasma, Titanium alloy, Stem cell

A successful outcome of joint replacement or dental restoration requires the development of a metal implant and of a biological facilitator that can promote bone-to-metal osseointegration. ${ }^{1)}$ Titanium alloys are widely used for bone

Received December 23, 2018; Accepted March 1, 2019

Correspondence to: Jeong Joon Yoo, MD

Department of Orthopedic Surgery, Seoul National University College of Medicine, 103 Daehak-ro, Jongno-gu, Seoul 03080, Korea

Tel: +82-2-2072-1994, Fax: +82-2-764-2718

E-mail: jjyos@snu.ac.kr reconstruction in orthopedic and dental surgery. These alloys work better than other metals because of their lightness, strength, toughness, high corrosion resistance, and low thermal conductivity. ${ }^{2)}$ Despite their wide implementation in currently used implants, surface activation of titanium remains a very active area of research. Findings suggest that surface modification may accelerate peri-implant bone formation. ${ }^{1,3)}$

Stem cells are a very active area of research in medicine. They can be classified by their ability to differentiate into different cell types. For orthopedic and dental surgery, 
Hong et al. Platelet-Rich Plasma Enhancing Osteogenic Differentiation of Adipose-Derived Stem Cells

Clinics in Orthopedic Surgery • Vol. 11, No. 3, $2019 \bullet$ www.ecios.org

researchers are mostly interested in mesenchymal stem cells, because they can differentiate into lineages of mesodermal tissues such as skeletal bone, tendons, cartilage, and fat. ${ }^{4,5)}$ In addition, they cause few ethical or legal problems, and they are more accessible. ${ }^{6}$ Bone marrow (BM) is a major source of mesenchymal stem cells that are relatively easy to harvest and simple to handle in vitro. ${ }^{5,7)}$ However, for clinical use, BM-derived stem cells may be detrimental because of the highly invasive extraction procedure and because mesenchymal stem cells differentiate less well as they age. ${ }^{8,9)}$ Adipose-derived stem cells (ADSCs) are a promising alternative to BM-derived stem cells as a source of mesenchymal stem cells. The easy and repeatable access to subcutaneous adipose tissue and simple isolation procedures provide clear advantages. ${ }^{4,10)}$

Platelet-rich plasma (PRP) can be used to deliver growth factors in high concentration to sites requiring augmented healing processes, including bone regeneration. ${ }^{11,12)}$ During peri-implant endosseous healing, a periimplant blood clot is responsible for osteoconduction. Integral to this process, PRP can act as osteoconductive material. It can also directly affect osteogenic cell migration. Although it may have wide clinical applications, the efficacy of PRP has yet to be established. A few in vitro and in vivo studies have reported the efficiency of PRP. ${ }^{13)}$

Our previous studies have demonstrated that a PRP-treated surface can increase early proliferation and late osteogenic differentiation of human osteoblasts and human bone-marrow stromal cells (hBMSCs). ${ }^{7,14)}$ Other studies have reported osteogenic differentiation of human adipose-derived stem cells (hADSCs) on a metal surface, especially on a titanium surface. ${ }^{2,6,15,16)}$ However, to the best of our knowledge, no studies have addressed whether hADSCs show better osteogenic differentiation on titanium-alloy surfaces and whether PRP pretreatment can promote this process.

In this study, we hypothesized that proliferation and osteogenic differentiation of hADSCs might occur on titanium alloys. We also hypothesized that the initial cell attachment and osteogenic process on titanium alloys might be influenced by whether the surface was pretreated with PRP. Thus, we intended to investigate whether PRP on a titanium alloy implant surface could affect initial cell attachment, cell proliferation, and osteogenic differentiation of hADSCs. To achieve this purpose, we analyzed the attachment, proliferation, and osteogenic differentiation of hADSCs on titanium alloys with or without PRP pretreatment.

\section{METHODS}

\section{PRP Preparation}

Allogenic leukocyte-depleted PRP was obtained from blood supernatants after 10 minutes of centrifugation at $150 \mathrm{~g}$ following removal of leukocytes. To achieve epidemiologically representative values of growth factors, PRP from three donors was mixed. The mean platelet counts in the PRP ranged from $800 / \mathrm{nL}$ to $1,100 / \mathrm{nL}$.

\section{Grit-Blasted Titanium Plug Preparation}

Titanium plugs (Titanium-6 Alumina-4 Vanadium, Ti$6 \mathrm{Al}-4 \mathrm{~V}, 7.7 \mathrm{~mm}$ in diameter, $2.8 \mathrm{~mm}$ in thickness) with rough surfaces were prepared by grit-blasting (TiGB) using an inhalation sanding machine (Zimmer Biomet Co., Warsaw, IN, USA) (Fig. 1). The surface roughness was generally $6.35 \mu \mathrm{m}$. These plugs were manufactured as screw-hole caps in an acetabular component for total hip arthroplasty. Plugs were washed and rendered hydrophilic by boiling for 5 minutes in $\mathrm{NH}_{4} \mathrm{OH}(25 \%): \mathrm{H}_{2} \mathrm{O}_{2}(30 \%)$ : $\mathrm{H}_{2} \mathrm{O}(1: 1: 5 \mathrm{v} / \mathrm{v})$ succeeded by meticulous cleansing in distilled water. All plugs were sonicated for 15 minutes in 95\% ethanol, rinsed with Dulbecco's Phosphate Buffered Saline (DPBS; Gibco-BRI, Gaithersburg, MD, USA) three times, and autoclaved for 15 minutes at $121^{\circ} \mathrm{C}$.

\section{PRP Pretreatment}

The TiGB plugs were placed individually at the bottom of 24-well plates, with $200 \mu \mathrm{L}$ of PRP added to each well. To adhere the platelets to the grit-blasted titanium surface, the plates were incubated at room temperature for 20 minutes. After the first incubation, further incubation at $37^{\circ} \mathrm{C}$ for 60 minutes was performed on a horizontal shaker (70 rpm). After a total of 80 minutes of contact, the plates were rinsed three times using phosphate-buffered
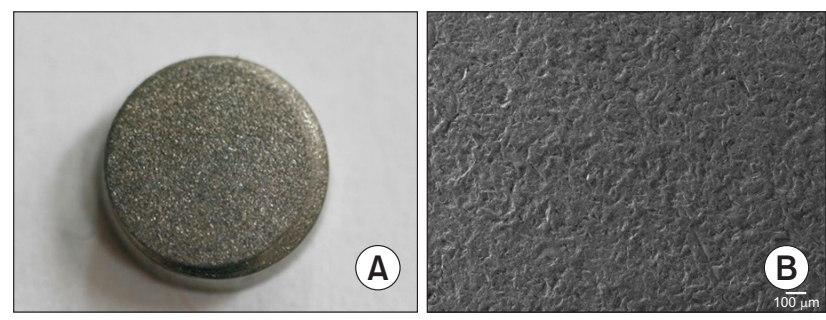

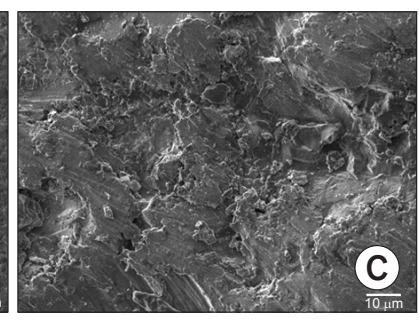

Fig. 1. (A) Morphology of grit-blasted titanium alloy (TiGB) plug. Macroscopic image (B) and scanning electron microscope image $(C)$ of the TiGB plug $(\times 60$ and $\times 1,000$, respectively). 
Hong et al. Platelet-Rich Plasma Enhancing Osteogenic Differentiation of Adipose-Derived Stem Cells

Clinics in Orthopedic Surgery • Vol. 11, No. 3, $2019 \bullet$ www.ecios.org

saline to eliminate loosely adherent platelets and residual PRP. Then, $200 \mu \mathrm{L}$ of Dulbecco's modified eagle medium (DMEM; Gibco, Invitrogen, Carlsbad, CA, USA) was added to each well of the plate followed by incubation at $37^{\circ} \mathrm{C}$. Calcium in the medium initiated the clotting cascade to form a fibrin network.

\section{Isolation and Culture of hADSCs}

The hADSCs were isolated from three donor's proximal lateral thigh subcutaneous fat tissue during surgery for total hip arthroplasty. We conducted this study in compliance with the principles of the Declaration of Helsinki. This study was approved by the Institutional Review Board of Seoul National University Hospital (IRB No. 1801125-917), which waived informed consent. Five grams of subcutaneous fat tissues were obtained from each donor. Extracted fat tissues were treated with $20 \mathrm{~mL}$ of DPBS and chopped with a sterilized pincette and surgical blade. These fat tissues were then treated with collagenase solution (fetal bovine serum-free media $20 \mathrm{~mL}+$ collagenase $0.015 \mathrm{~g}$ ). The hADSCs were then isolated by serial centrifugation after treatment with red blood cell lysis buffer. Isolated hADSCs were cultured and expanded up to passage 4 with DMEM and Ham's F12 medium (HAM-F12; Cultilab, Campinas, Brazil; 1:1 mixture) as growth media.

\section{Attachment and Proliferation of hADSCs}

The hADSCs were seeded in two groups: on TiGB and on TiGB coated with PRP (TiGB-PRP). For osteogenic differentiation of hADSCs, supplements were added into the culture media, including $50 \mu \mathrm{g} / \mathrm{mL}$ ascorbic acid, $10 \mathrm{mM}$ glycerol-2-phosohate, and $0.1 \mu \mathrm{M}$ dexamethasone. The osteogenic medium was replaced twice a week for 14 or 21 days. The number of hADSCs was measured after 4 hours, 3 days, and 7 days of culture using Cell Counting Kit- 8
(Dojindo, Tokyo, Japan) according to the manufacturer's instructions to analyze cell adhesion and proliferation.

\section{Osteogenic Differentiation}

Osteogenesis of hADSCs was measured by using an alkaline phosphatase (ALP) activity assay on days 7 and 14, and a calcium assay on days 14 and 21. ALP diagnostic kit (no. 245; Sigma-Aldrich, St. Louis, MO, USA) was used to measure the ALP activities, on days 7 and 14. Cultured hADSCs were treated with $0.1 \%$ Triton in water to obtain cell lysate. Then $10 \mu \mathrm{L}$ of cell lysate was introduced per well in a 24-well plate, followed by an addition of $50 \mu \mathrm{L}$ of ALP buffer (no. A-9226, Sigma-Aldrich) to each well. The plate was stirred for 30 minutes at room temperature. Then $50 \mu \mathrm{L}$ of $0.05 \mathrm{~N} \mathrm{NaOH}$ was added, whereafter the plate was stirred for 5 minutes. Finally, VERSAmax Tunable Microplate Reader (Molecular Devices, Sunnyvale, CA, USA) was used to measure the absorbance of $\mathrm{p}$ nitrophenol, the reaction product, at $405 \mathrm{~nm}$. ALP activity was calculated from the absorbance by using a reference sample, p-nitrophenol standard (no. N-7660, SigmaAldrich). The recorded activities were in $\mu \mathrm{mol}$ p-nitrophe$\mathrm{nol} / \mathrm{min} / \mathrm{mg}$ of protein per well. Calculated ALP activities were standardized against total protein content, which was measured using BioRad Protein assay kit (kit II, BIO-RAD Laboratories).

On days 14 and 21, QuantiChrom calcium assay kit (DICA-500; BioAssay Systems, Hayward, CA, USA) was used to measure calcium deposit amounts. Cell layers were rinsed using $\mathrm{Ca}^{2+}$ - and $\mathrm{Mg}^{2+}$-free phosphatebuffered saline, which was then solubilized by $0.6 \mathrm{~N} \mathrm{HCl}$. Each sample $(5 \mu \mathrm{L})$ was introduced into a 24 -well plate, followed by an addition of $200 \mu \mathrm{L}$ of working agent to each well. The mixture was left to react for 3 minutes at room temperature. VERSAmax Tunable Microplate Reader was

Table 1. Sequences of Primers Used for RT-PCR Analysis and Corresponding Size of Attempted PCR Products

\begin{tabular}{|c|c|c|c|c|}
\hline Target transcript & Primer sequence & Annealing temperature $\left({ }^{\circ} \mathrm{C}\right)$ & PCR cycle & Predicted size (bp) \\
\hline ALP & $\begin{array}{l}\text { (F) 5'-TGGAGCTTCAGAAGCTCAACACCA-3' } \\
\text { (R) 5'-ATCTCGTTGTCTGAGTACCAGTCC-3' }\end{array}$ & 61 & 30 & 454 \\
\hline Osteocalcin & $\begin{array}{l}\text { (F) 5'-ATGAGAGCCCTCACACTCCTCGCCCTA-3' } \\
\text { (R) 5'-GACCGGGCCGTAGAAGCGCCGATAGG-3' }\end{array}$ & 68 & 30 & 300 \\
\hline Type I collagen & $\begin{array}{l}\text { (F) 5'-CTCGAGGTGGACACCACCCT-3' } \\
\text { (R) 5'-CAGCTGGATGGCCACATCGG-3' }\end{array}$ & 62 & 30 & 372 \\
\hline GAPDH & $\begin{array}{l}\text { (F) 5'-ATTGTTGCCATCAATGACCC-3' } \\
\text { (R) 5'-AGTAGAGGCAGGGATGATGTT-3' }\end{array}$ & 55 & 28 & 546 \\
\hline
\end{tabular}

RT-PCR: reverse transcription polymerase chain reaction, F: forward, R: reverse, ALP: alkaline phosphatase, GAPDH: glyceraldehyde-3-phosphate dehydrogenase. 
Hong et al. Platelet-Rich Plasma Enhancing Osteogenic Differentiation of Adipose-Derived Stem Cells

Clinics in Orthopedic Surgery • Vol. 11, No. 3, $2019 \bullet$ www.ecios.org

used to measure the absorbance of the reactant at $612 \mathrm{~nm}$. Calcium standard $\left(1 \mathrm{~mL} 20 \mathrm{mg} / \mathrm{dL} \mathrm{Ca}^{2+}\right)$ was prepared as reference samples. From the absorbance, the amount of calcium deposit was derived in terms of $\mu \mathrm{g} / \mathrm{mL}$ following the manufacturer's instruction.

\section{Reverse Transcription Polymerase Chain Reaction Analysis}

Total RNAs were extracted using TRIzol reagent (Invitrogen) on culture days 14 and 21. Single strand cDNA synthesis was performed using First Strand cDNA Synthesis kits (Invitrogen). These cDNAs were mixed with PCR cocktail (AccuPower PCR Premix, Bioneer, Daejeon, Korea) and $0.5 \mu \mathrm{M}$ of both forward and reverse primers (Table 1). Amplification was performed on a GeneAmp PCR system thermocycler (Applied Biosystems, Foster City, CA, USA). These PCR products were separated by electrophoresis on $1 \%(\mathrm{w} / \mathrm{v})$ agarose gels and visualized by ethidium bromide staining.

\section{Field-Emission Scanning Electron Microscope Observation}

The microscopic morphology of TiGB with or without PRP was examined with a field-emission scanning electron microscope (FE-SEM) using a JSM-7401F apparatus (JEOL, Tokyo, Japan) on days 1 and 7. After removal of the medium, the obtained plugs were rinsed with DPBS. Then using $1,000 \mu \mathrm{L}$ of $2 \%$ glutaraldehyde (no. G-5882, SigmaAldrich), the plugs were fixed at $4^{\circ} \mathrm{C}$ for 2 hours. The plugs were subsequently washed using DPBS and dried by immersing in serially incrementing ethyl alcohol concentrations $(70 \%-100 \%)$ succeeded by vacuum drying. The specimens were coated with gold-palladium alloy and mounted on aluminum stubs to be examined under FE-SEM.

\section{Statistical Analysis}

Each experiment was done in triplicate with five samples per group, amounting at fifteen samples for each group. Data are presented as average \pm standard deviation. A Mann-Whitney test was used to compare the TiGB and TiGB-PRP plugs. Differences were considered statistically significant if $p<0.05$.

\section{RESULTS}

Fig. 1 shows the morphology of grit-blasted titanium alloy. Macroscopic images of TiGB showed that the surface was not smooth, based on scattered reflection of light (Fig. 1A). FE-SEM showed a rough surface with a relatively uniform pattern of grooves, ridges, hills, ranges, valleys, and crevices (Fig. 1B and C).

Fig. 2 shows adhered hADSCs after 1 day and proliferated cells after 7 days of culture on TiGB and TiGBPRP. Adhered hADSCs were observed on day 1 of culture. Cells were scattered on TiGB, and the number of cells was relatively low (Fig. 2A). On TiGB-PRP, adhered hADSCs stretched over the surface, and the number of cells was relatively high (Fig. 2C). More cells and fibrin network

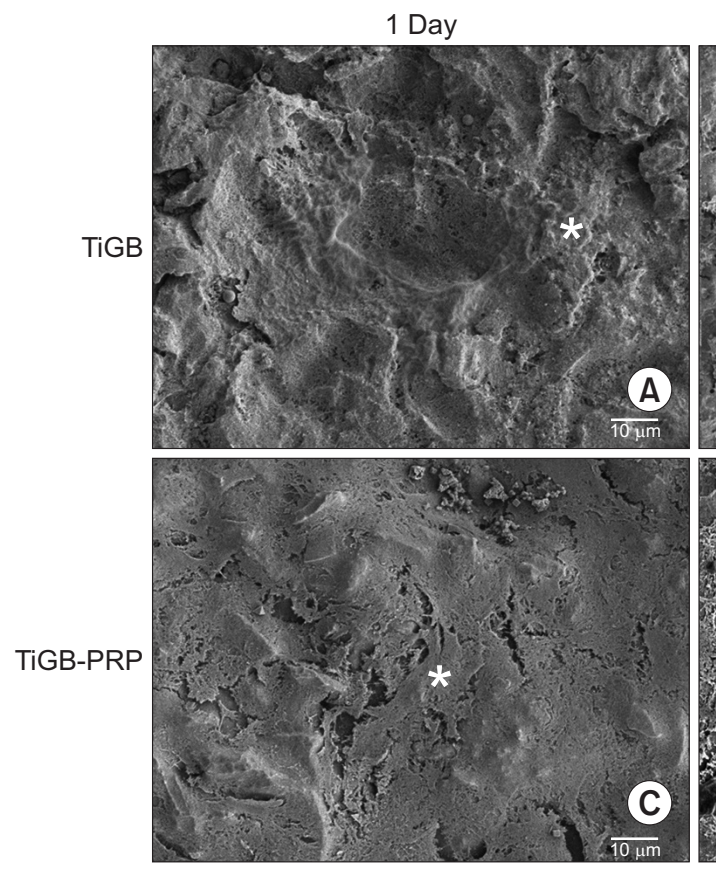

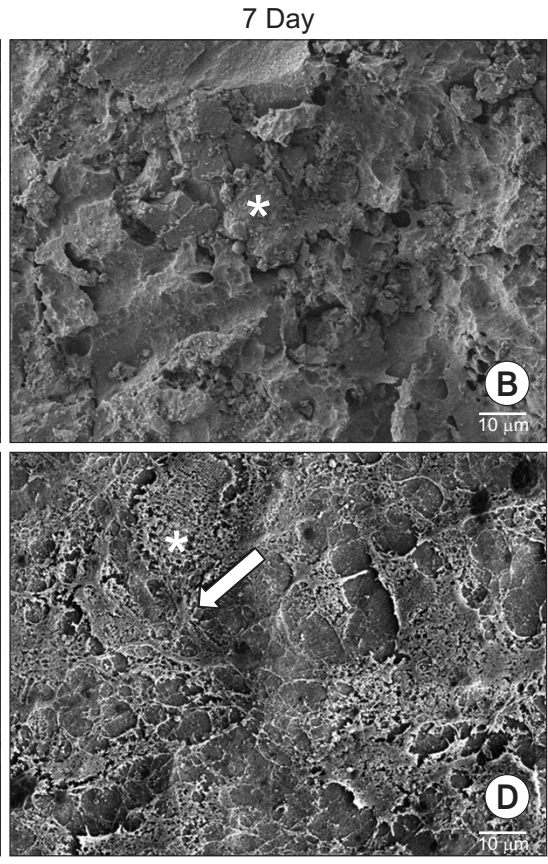

Fig. 2. Field-emission scanning electron microscope images $(\times 1,000$ magnification) of adhered human adiposederived stem cells after 1 day and 7 days of culture on TiGB $(A, B)$ and TiGB$\operatorname{PRP}(C, D) .(C)$ and (D) appeared different because fibrin network covered the raw surface of TiGB-PRP. Asterisks: adhered cells, arrow: fibrin network. TiGB: gritblasted titanium alloy, PRP: platelet-rich plasma. 
were observed on day 7 of culture. There were many more cells on the TiGB-PRP on day 7 than there had been on day 1 . In addition, proliferated cells were surrounded and stabilized by extensive three-dimensional fibrin networks (Fig. 2B and D).

Cell attachment was estimated after 4 hours of culture on TiGB and TiGB-PRP. Cell attachment on TiGBPRP was 2.4-fold that on TiGB (Fig. 3A). On day 3, the numbers of cells attached on both plugs decreased slightly, followed by an increase thereafter. Cell proliferation on day 7 was significantly higher in TiGB-PRP than in TiGB $(p=0.016)$ (Fig. 3B). More than twice as many cells were observed on TiGB-PRP plugs after 7 days of culture than there were on day 1 .

Osteogenic differentiation of hADSCs was evaluated by measuring ALP activity and calcium content. After 7 and 14 days of differentiation, ALP activities were significantly higher in TiGB-PRP $(p=0.008)$ than in TiGB (Fig. $4 \mathrm{~A})$. Similarly, calcium contents were more than three times higher in TiGB-PRP $(p=0.008)$ than in TiGB (Fig. 4B) after 14 and 21 days of differentiation.

Reverse transcription polymerase chain reaction
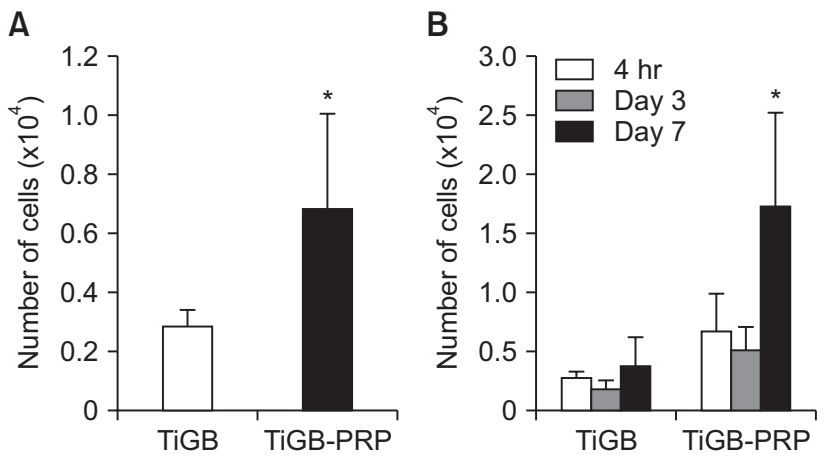

Fig. 3. Attachment and proliferation of human adipose-derived stem cells (hADSCs). (A) Attachment of hADSCs on TiGB and TiGB-PRP after 4 hours of culture. (B) Proliferation of hADSCs on TiGB and TiGB-PRP. TiGB: gritblasted titanium alloy, PRP: platelet-rich plasma. ${ }^{*} p<0.05$.
(RT-PCR) analysis demonstrated that ALP, osteocalcin, type I collagen mRNA were expressed higher in the TiGBPRP group than in the TiGB group after 14 days of differentiation (Fig. 5A). On the other hand, the expression of ALP, osteocalcin, type I collagen mRNA was expressed similar between the TiGB and TiGB-PRP groups after 21 days of differentiation (Fig. 5B).

\section{DISCUSSION}

Bone-marrow stromal cells (BMSCs) and ADSCs are widely studied for tissue-engineered bone regeneration. $\mathrm{BM}$ was the initial source claimed to possess mesenchymal stem cells. Yet the BM may be considered unfavorable for clinical use because of the invasive extraction method and decrease of mesenchymal stem cells in both number and differentiation potential with age. ${ }^{17)}$ Instead, ADSCs have been proven to be a promising alternative source of mesenchymal stem cells. Although mesenchymal stem cells derived from $\mathrm{BM}$ have a shorter culture period than do ADSCs, there are no significant differences in morphol-
A

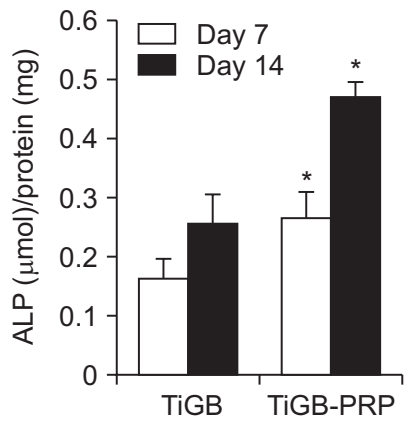

B

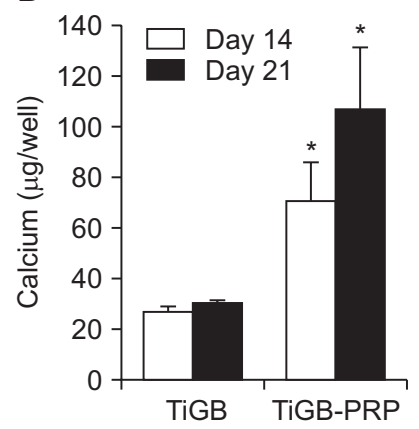

Fig. 4. Quantitative results of osteogenic differentiation of human adiposederived stem cells (hADSCs) on plugs. (A) Alkaline phosphatase (ALP) activities of hADSCs after 7 and 14 days of culture. (B) Calcium content of hADSCs after 14 and 21 days of culture. TiGB: grit-blasted titanium alloy, PRP: platelet-rich plasma. ${ }^{*} p<0.05$.
A

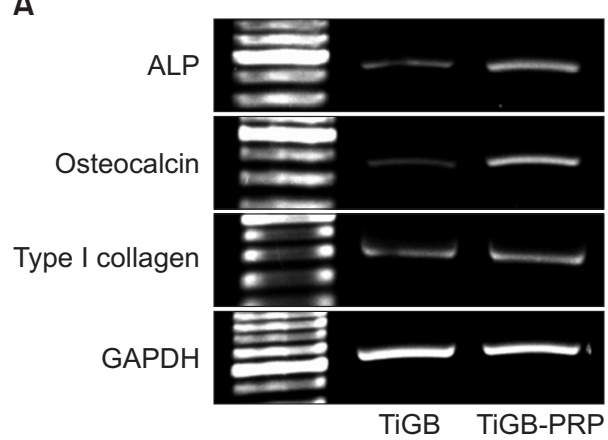

B

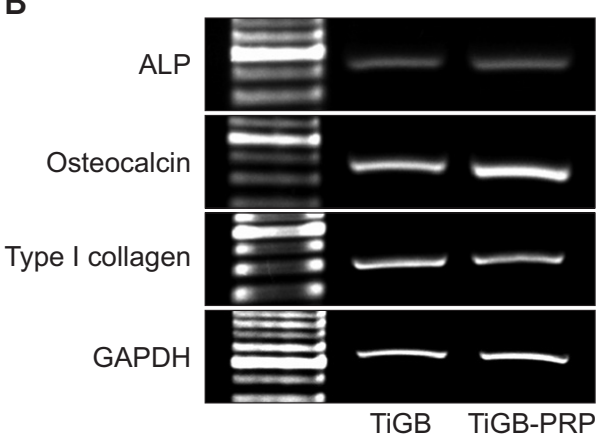

Fig. 5. Expression of alkaline phosphatase (ALP), osteocalcin, and type I collagen mRNA after osteogenic differentiation for 14 days (A) and 21 days (B). GAPDH: glyceraldehyde-3-phosphate dehydrogenase, TiGB: grit-blasted titanium alloy, PRP: platelet-rich plasma. 
Hong et al. Platelet-Rich Plasma Enhancing Osteogenic Differentiation of Adipose-Derived Stem Cells

Clinics in Orthopedic Surgery • Vol. 11, No. 3, 2019• www.ecios.org

ogy, immunophenotype, or success rate of isolating the mesenchymal stem cells between BMSCs and ADSCs. ${ }^{18,19)}$ Based on findings of previous studies, we chose hADSCs rather than hBMSCs that tend to be obtained by more invasive methods from patients.

Platelets are known to activate the coagulation cascade that can prevent excessive bleeding, and plateletderived growth factors and cytokines help wound healing. ${ }^{20)}$ PRP delivers these growth factors and cytokines in high concentration to sites that need an augmented healing process, which in turn will promote mesenchymal cell migration, proliferation, and differentiation. ${ }^{11,12,21)}$ As a result, many clinicians have used high concentrations of platelets to treat injuries or degenerative diseases. ${ }^{22)}$ Shin et al. ${ }^{7)}$ showed better proliferation and osteogenic differentiation of hBMSCs in tissue culture plates with PRP. In agreement with previous findings, our study also showed better proliferation and osteogenic differentiation of hADSCs in PRP-pretreated grit-blasted titanium alloys (TiGB-PRP).

Titanium alloys, including Ti-6Al-4V, are widely used for artificial metal implantation surgery because of their excellent intrinsic properties, such as lightness, strength, toughness, high corrosion resistance, and low thermal conductivity. ${ }^{2)}$ To maximize the osseointegration effect, it is necessary to roughen the implant surface. Several methods including grit-blasting have been used to achieve a rough surface. The degree of surface roughness determines the amount of bone ongrowth and ingrowth around the implant. ${ }^{23)}$ The roughness of the metal surface is expressed with $R_{a}$ (average roughness value). Previous studies have used grit-blasted titanium alloys, showing $\mathrm{R}_{\mathrm{a}}$ between 0.32 and $11 \mu \mathrm{m} .^{24,25)}$ TiGB used in this study was treated to achieve $R_{a}$ of $6.35 \mu \mathrm{m}$, which coincided with the acceptable range shown in previous studies.

In this study, the initial attachment of hADSCs to the metal surface was 2.4 times higher on TiGB-PRP than on TiGB, although the difference was not statistically significant $(p=0.056)$. FE-SEM images taken on the first day also showed more cells distributed on TiGB-PRP (Fig. 2A and B). Although the statistical significance was weak, this finding suggests that PRP pretreatment on metal surfaces could improve cell attachment. Some studies have shown that platelet integrins can provide an environment suitable for attachment, migration, and differentiation of cells. ${ }^{26)}$ After 7 days of culture, the number of cells observed on TiGB-PRP had increased significantly more than that in $\operatorname{TiGB}(p=0.016)$. The difference between the two groups at the beginning was only 2.4 times. However, by the 7 th day, it had increased to 4.5 times. The only difference between the two groups was the presence or absence of
PRP. This suggests that PRP can stimulate cells to proliferate rapidly. The number of cells on the 3rd day showed a slight decrease in both TiGB and TiGB-PRP groups; cells attached around the implant might have stabilized for 3 days, and some cells might have fallen off from the implant surface.

The in vitro osteogenic differentiation of mesenchymal stem cells is divided into three stages. In the first stage, from day 1 to day 4, the number of cells peak. The second stage, from day 5 to day 14 , is characterized by early cell differentiation, marked by transcription and protein expression of ALP. In the third stage, from day 15 to day 28, rapid turnover of osteopontin and osteocalcin with subsequent calcium and phosphate deposition occurs. ${ }^{27)}$ In this study, the activity of ALP in TiGB-PRP was significantly higher than that in TiGB on the 7th day and the 14th day $(p=0.008)$. The amount of calcium was also significantly higher $(p=0.008)$ in TiGB-PRP on the 14th day and the 21 st day, indirectly showing that both early and late stages of osteogenic differentiation were more actively promoted by pretreated PRP. We also measured the level of osteogenic gene expression of ALP, osteocalcin, and type I collagen using RT-PCR analysis. ALP, osteocalcin, and type I collagen mRNA were markedly higher on the 14th day but the expressions on the 21st day were not significantly different. The mRNA is a temporal precedent product rather than the ALP activity or calcium formation, which is the final result of gene expression. Since RT-PCR is a test for mRNA, there was no difference in mRNA expression on the 21st day, although there was a difference between the two groups on the 14th day.

In clinical settings, metal implants are widely used to fill and reconstruct large bone defects. ${ }^{28-30)}$ The more bone forms around metal implants, the more successful a reconstruction operation will be. A cell adjuvant tissueengineered approach may be an option to improve metal implant-associated bone reconstruction. The hADSCs, a source of mesenchymal stem cells, can be obtained by simple liposuction from the patient and PRP can be extracted from the blood of the same patient. Both hADSCs and PRP have autologous characteristics. Thus, they are free from ethical concerns or risk of disease transmission. With these advantages, reconstructive surgery can be performed using an autologous PRP pretreatment on a grit-blasted titanium alloy with autologous hADSCs in a staged operation. However, our hypothesis has yet to be supported by an in vivo study. To further verify our findings, the effect of hADSCs on PRP-pretreated titanium alloy on bone-defect regeneration in vivo needs to be investigated in the future.

Our results demonstrated that a hADSCs-based ap- 
proach could be used for tissue-engineered peri-implant bone formation and that PRP pretreatment on a titanium plug could increase proliferation and osteogenic differentiation of hADSCs. PRP from autologous blood and hADSCs taken from the same patient by simple liposuction may improve bone-defect reconstruction using titaniumalloy implantation.

\section{CONFLICT OF INTEREST}

No potential conflict of interest relevant to this article was reported.

\section{ACKNOWLEDGEMENTS}

We would like to acknowledge the support of National Research Foundation (NRF) funded by the Ministry of Education, Science and Technology, Republic of Korea, through their grant (2015-01004099) of the Basic Science Research Program. The study was also supported by a grant (0420160370) from Seoul National University Hospital Research Fund.

\section{REFERENCES}

1. Han SK, Chang YJ, Kim YS, Lee JY, Lim YW. Effect of surface modification on biomechanical properties of titanium alloy Ti6Al4V. Tissue Eng Regen Med. 2010;7(3):338-43.

2. Malec K, Goralska J, Hubalewska-Mazgaj M, et al. Effects of nanoporous anodic titanium oxide on human adipose derived stem cells. Int J Nanomedicine. 2016;11:5349-60.

3. Lee MH, Oh NS, Lee SW, Kang JH, Lee SC, Leesungbok R. Enhancement of dynamic wettability, cell adhesion, and alkaline phosphatase activity of primary cells on titanium substrata with combined surface topographies of microgrooves and acid-etched roughness. Tissue Eng Regen Med. 2010;7(5):501-12.

4. Schaffler A, Buchler C. Concise review: adipose tissue-derived stromal cells--basic and clinical implications for novel cell-based therapies. Stem Cells. 2007;25(4):818-27.

5. Hempel U, Muller K, Preissler C, et al. Human bone marrow stromal cells: a reliable, challenging tool for in vitro osteogenesis and bone tissue engineering approaches. Stem Cells Int. 2016;2016:7842191.

6. Nae S, Bordeianu I, Stancioiu AT, Antohi N. Human adipose-derived stem cells: definition, isolation, tissueengineering applications. Rom J Morphol Embryol. 2013;54(4):919-24.

7. Shin SH, Yoo JJ, Kim HN, Nam J, Kim HJ. Enhanced cellular responses of human bone marrow stromal cells cultured on pretreated surface with allogenic platelet-rich plasma. Connect Tissue Res. 2012;53(4):318-26.

8. D'Ippolito G, Schiller PC, Ricordi C, Roos BA, Howard GA. Age-related osteogenic potential of mesenchymal stromal stem cells from human vertebral bone marrow. J Bone Miner Res. 1999;14(7):1115-22.

9. Rao MS, Mattson MP. Stem cells and aging: expanding the possibilities. Mech Ageing Dev. 2001;122(7):713-34.
10. Gir P, Oni G, Brown SA, Mojallal A, Rohrich RJ. Human adipose stem cells: current clinical applications. Plast Reconstr Surg. 2012;129(6):1277-90.

11. Mehta S, Watson JT. Platelet rich concentrate: basic science and current clinical applications. J Orthop Trauma. 2008;22(6):432-8.

12. van den Dolder J, Mooren R, Vloon AP, Stoelinga PJ, Jansen JA. Platelet-rich plasma: quantification of growth factor levels and the effect on growth and differentiation of rat bone marrow cells. Tissue Eng. 2006;12(11):3067-73.

13. Song HR, Bae JH, Park JH, et al. The effect of platelet rich plasma on osteogenesis in a long bone segmental defect : is the platelet rich plasma effective for bone reconstruction? Tissue Eng Regen Med. 2010;7(4):395-400.

14. Lee JH, Nam J, Nam KW, Kim HJ, Yoo JJ. Pre-treatment of titanium alloy with platelet-rich plasma enhances human osteoblast responses. Tissue Eng Regen Med. 2016;13(4):33542.

15. Zanicotti DG, Duncan WJ, Seymour GJ, Coates DE. Effect of titanium surfaces on the osteogenic differentiation of human adipose-derived stem cells. Int J Oral Maxillofac Implants. 2018;33(3):e77-87.

16. Gastaldi G, Asti A, Scaffino MF, et al. Human adiposederived stem cells (hASCs) proliferate and differentiate in osteoblast-like cells on trabecular titanium scaffolds. J Biomed Mater Res A. 2010;94(3):790-9.

17. Stolzing A, Jones E, McGonagle D, Scutt A. Age-related changes in human bone marrow-derived mesenchymal stem cells: consequences for cell therapies. Mech Ageing Dev. 2008;129(3):163-73.

18. Izadpanah R, Trygg C, Patel B, et al. Biologic properties of mesenchymal stem cells derived from bone marrow and adipose tissue. J Cell Biochem. 2006;99(5):1285-97. 
19. Kern S, Eichler H, Stoeve J, Kluter H, Bieback K. Comparative analysis of mesenchymal stem cells from bone marrow, umbilical cord blood, or adipose tissue. Stem Cells. 2006;24(5):1294-301.

20. Sundman EA, Cole BJ, Fortier LA. Growth factor and catabolic cytokine concentrations are influenced by the cellular composition of platelet-rich plasma. Am J Sports Med. 2011;39(10):2135-40.

21. Sanchez AR, Sheridan PJ, Kupp LI. Is platelet-rich plasma the perfect enhancement factor? A current review. Int J Oral Maxillofac Implants. 2003;18(1):93-103.

22. de Vos RJ, Weir A, van Schie HT, et al. Platelet-rich plasma injection for chronic Achilles tendinopathy: a randomized controlled trial. JAMA. 2010;303(2):144-9.

23. Buser D, Schenk RK, Steinemann S, Fiorellini JP, Fox CH, Stich H. Influence of surface characteristics on bone integration of titanium implants: a histomorphometric study in miniature pigs. J Biomed Mater Res. 1991;25(7):889-902.

24. Lakstein D, Kopelovitch W, Barkay Z, Bahaa M, Hendel D, Eliaz N. Enhanced osseointegration of grit-blasted, $\mathrm{NaOH}$ treated and electrochemically hydroxyapatite-coated $\mathrm{Ti}$ 6Al-4V implants in rabbits. Acta Biomater. 2009;5(6):2258-
69.

25. Wu Y, Zitelli JP, TenHuisen KS, Yu X, Libera MR. Differential response of Staphylococci and osteoblasts to varying titanium surface roughness. Biomaterials. 2011;32(4):95160.

26. Garcia AJ. Get a grip: integrins in cell-biomaterial interactions. Biomaterials. 2005;26(36):7525-9.

27. Huang Z, Nelson ER, Smith RL, Goodman SB. The sequential expression profiles of growth factors from osteoprogenitors [correction of osteroprogenitors] to osteoblasts in vitro. Tissue Eng. 2007;13(9):2311-20.

28. Dwivedi C, Gokhale S, Khim HG, Oh JK, Shon WY. Acetabular defect reconstruction with trabecular metal augments: study with minimum one-year follow-up. Hip Pelvis. 2017;29(3):168-75.

29. Janecka IP. New reconstructive technologies in skull base surgery: role of titanium mesh and porous polyethylene. Arch Otolaryngol Head Neck Surg. 2000;126(3):396-401.

30. Robinson Y, Tschoeke SK, Kayser R, Boehm H, Heyde CE. Reconstruction of large defects in vertebral osteomyelitis with expandable titanium cages. Int Orthop. 2009;33(3):7459. 